\title{
Economía de plataformas y turismo en España a través de Airbnb*
}

\section{Platform economy and tourism in Spain: The Airbnb case}

\author{
Armando Ortuño \\ Universidad de Alicante \\ Juan Luis Jiménez \\ Universidad de Las Palmas de Gran Canaria
}

\section{Resumen}

Las plataformas online de alquiler de viviendas turísticas han experimentado un crecimiento destacado en España durante el último lustro, al igual que alrededor del mundo. Ello ha modificado en buena medida las pautas de consumo alojativo turístico y, en adaptación a ello, la normativa de aplicación. En el presente trabajo realizamos una aproximación cualitativa y descriptiva a la expansión de Airbnb en España, especialmente en los dos modelos de desarrollo turísticos predominantes en España (cultural en centros urbanos de grandes ciudades y residencial de «sol y playa»), al análisis de la literatura que evalúa sus efectos y a los cambios regulatorios aplicados en cada Comunidad Autónoma. Finalmente, se propone una metodología de second-best que podría reducir los efectos adversos que la regulación actual está generando.

Palabras clave: Airbnb, regulación, economía de plataformas.

Clasificación JEL: L83, Z31, Z38.

\begin{abstract}
On-line touristic rent platforms have experienced a relevant growth in Spain (and all over the world) during the last five years. These platforms have modified to a large extent the patterns of tourist accommodation and, in adaptation to this, the regulations. In the present paper we conduct a qualitative and descriptive approach to the expansion of Airbnb in Spain, especially in the two predominant tourism development models in Spain (cultural in urban centers of large cities and residential «sun and beach»). We also describe the academic literature that evaluates its effects and the regulatory changes applied in each Autonomous Community. Finally, a secondbest methodology is proposed that could reduce the adverse effects that the current regulation is generating.
\end{abstract}

Keywords: Airbnb, regulation, platform economy.

JEL classification: L83, Z31, Z38.

* Los autores agradecen la financiación recibida por FEDEA para realizar el proyecto de investigación titulado «Las viviendas turísticas ofertadas por plataformas online: estado de la cuestión y evaluación de efectos en turismo y alquileres en España», del que se ha nutrido el presente artículo. Armando Ortuño desea agradecer también la financiación para el trabajo «Diagnóstico de potencialidades plataformas P2P de alquiler de uso turístico. Municipios turístico-residenciales de la Costa Blanca», recibida de la Excma. Diputación Provincial de Alicante. No obstante, cualquier error es entera responsabilidad de los autores. 


\section{Motivación}

La conjunción en el tiempo del auge de las plataformas online de economía colaborativa en el sector del alojamiento turístico, junto con la modificación en 2013 de la Ley de arrendamientos urbanos (LAU), está alterando las condiciones de los mercados turísticos, modificando los períodos de oferta y demanda, ampliando los destinos casi a cualquier localización posible y modificando las redes y sistemas de contratación de los servicios (Ortuño et al., 2018b).

Efectivamente, uno de los fenómenos impulsores de estas importantes alteraciones se encuentra en el desarrollo de la economía colaborativa definida por la Comisión Europea como «aquellos modelos de negocio en los que se facilitan actividades mediante plataformas colaborativas que crean un mercado abierto para el uso temporal de mercancías o servicios ofrecidos a menudo por particulares. La economía colaborativa implica a tres categorías de agentes: I) prestadores de servicios que comparten activos, recursos, tiempo y/o competencias -pueden ser particulares que ofrecen servicios de manera ocasional («pares») o prestadores de servicios que actúen a título profesional («prestadores de servicios profesionales»); II) usuarios de dichos servicios; y III) intermediarios que -a través de una plataforma en líneaconectan a los prestadores con los usuarios y facilitan las transacciones entre ellos («plataformas colaborativas»). Por lo general, las transacciones de la economía colaborativa no implican un cambio de propiedad y pueden realizarse con o sin ánimo de lucro» (COM, 2016, pág. 3).

Tal y como señalan Ortuño y Jiménez (2019), la economía colaborativa ha tenido su expansión a partir del desarrollo y la democratización en el uso de internet y las aplicaciones necesarias para conectar a ambos lados del mercado (two-sided markets). Este cambio en el modelo de consumo, la propiedad y las relaciones sociales ha tenido su expansión hacia el mercado del alojamiento.

Junto al fenómeno de la economía colaborativa, se ha añadido la citada modificación de la Ley de Arrendamientos Urbanos (LAU) mediante la Ley 4/2013, de 4 de junio, de medidas de flexibilización y fomento del mercado de alquiler de viviendas que, entre otras cosas, supuso la exclusión del ámbito de regulación de la LAU de un determinado tipo de arrendamiento. En concreto, la exclusión se regula con la introducción de una nueva letra e) dentro del artículo 5, relativa a «la cesión temporal de uso de la totalidad de una vivienda amueblada y equipada en condiciones de uso inmediato, comercializada o promocionada en canales de oferta turística y realizada con finalidad lucrativa, cuando esté sometida a un régimen específico, derivado de su normativa sectorial».

Como consecuencia de este cambio, el alquiler de viviendas que cumpla todos los requisitos del artículo 5.e), queda regulado a través de la normativa turística, competencia que pertenece, en exclusiva, a las Comunidades Autónomas. Estas han comenzado a legislar sobre la materia de manera dispar, lo que incluso ha dado lugar a la nulidad determinadas normativas autonómicas y al conflicto acusado entre estas legislaciones y los organismos de competencia tanto autonómicos como estatales (casos de Canarias, Madrid o Cataluña, entre otros). 
Según Ortuño et al. (2018a), el crecimiento del número de turistas en España durante los últimos años está provocando una serie de reacciones y debate social sobre cuál debería ser el modelo de gestión turística del país, centrándose buena parte de ese debate en los impactos y regulación de los arrendamientos de estas viviendas de usos turísticos ofertadas a través de plataformas tecnológicas.

Sin duda, una de las principales plataformas que ha propiciado el auge de la economía colaborativa en el sector del alojamiento colaborativo es Airbnb. Desde su creación en 2008, el crecimiento de la actividad de la plataforma se ha disparado en todo el mundo de forma que, en diciembre de 2016, disponía de más de 2 millones de viviendas para alquilar en 34.000 ciudades de 191 países y 60 millones de consumidores. En 2016, Airbnb se situaba como la cuarta plataforma mundial, indiscutiblemente la primera en su sector, con una valoración por encima de los 22.000 millones de euros (Centre for Global Enterprise, 2016).

La plataforma y, de igual forma el resto de sus principales competidoras, está abierta para uso particular y profesional y dentro de ella se pueden alquilar viviendas, habitaciones, hoteles, reservar servicios denominados de bed and breakfast o apartamentos de vacaciones. Los servicios incluidos pueden variar desde el mero alojamiento hasta guías turísticas, planificación del viaje, pasando por la mayor parte de los servicios hoteleros (limpieza, desayunos, etc.).

Uno de los elementos más destacados de su actividad es la ausencia de alojamientos en propiedad, lo que le aleja del tradicional sistema de inmobiliarias u hoteles, sino que, como se ha descrito, se trata de una plataforma que pone en contacto la oferta y la demanda y que, por ello, obtiene unos ingresos asimilables a los de la clásica comisión por transacción (Ortuño et al., 2018a). En definitiva, las plataformas online como Airbnb están cambiando los modelos tradicionales de acceso al alojamiento turístico (Jimeno et al., 2018).

En este marco, el presente trabajo realizará una aproximación cualitativa al desarrollo de las plataformas online aplicadas a viviendas turísticas en España, centrados en Airbnb por ser la de mayor relevancia. Para ello, y tras esta introducción, se resumen algunas de las principales referencias académicas existentes sobre el sector (sección 2) y se describe cuáles han sido las principales actuaciones legislativas de las Comunidades Autónomas en España para afrontar este fenómeno (sección 3). La sección cuarta muestra la evolución de la oferta alojativa de Airbnb en el país, para posteriormente concluir con una serie de conclusiones y recomendaciones de actuación que podrían mejorar la eficiencia en la gestión municipal de estas actividades, fundamentalmente basadas en la disparidad de criterios y los efectos negativos que, presumiblemente, las restricciones de la competencia puedan tener sobre este sector. 


\section{2. ¿Qué efectos se han cuantificado sobre el desarrollo de Airbnb?}

La literatura académica que analiza los efectos de la irrupción de plataformas como Airbnb es relativamente reciente e incipiente. Como resumen Ortuño y Jiménez (2019), la mayoría de trabajos (además de los documentos de estudio realizados por alguna de las partes interesadas) son empíricos y centrados en el mercado de Estados Unidos, donde la expansión de esta empresa ha sido mayor y más rápida que en el resto del mundo.

Hasta donde conocemos, no existen estudios que analicen si el tamaño del mercado turístico varía ante la llegada de estos operadores, salvo el propio Ortuño y Jiménez (2019). En este documento, realizado para 38 ciudades españolas que acaparan más del 14 por 100 de la población total, apuntan a que no hay un efecto homogéneo en todas las ciudades y, en concreto, resaltan que el número de turistas aumenta principalmente en las grandes ciudades.

Uno de los principales efectos podría encontrarse también sobre las empresas ya instaladas en el sector, particularmente hoteles. Tal y como apuntan Zervas et al. (2017), en este caso, el nuevo operador se muestra como un competidor, de forma que afecta menos a hoteles de mayor categoría y precio, al contrario que a los hoteles independientes frente a los que pertenecen a cadenas hoteleras.

En cuanto a los efectos que la entrada de Airbnb tiene sobre el mercado inmobiliario, los artículos de Sheppard y Udell (2016), Horn y Merante (2017) y Barron, Kung y Proserpio (2017), justifican impactos de incremento en precios de alquileres y precios de venta de viviendas. Todos estos trabajos, como antes mencionamos, están centrados en el mercado de Estados Unidos.

El Cuadro 1, extraído de Ortuño y Jiménez (2019), muestra un breve resumen de esta literatura y sus principales conclusiones.

En concreto, y para el caso español, se dispone de los artículos realizados por Gutiérrez et al. (2017) y Eugenio-Martín et al. (2019), quienes se centran en la relación espacial entre la oferta alojativa de Airbnb y los hoteles. El primer caso utiliza datos de la ciudad de Barcelona, mientras que el segundo aplica a los mercados insulares canarios. El análisis de la oferta permite a Gutiérrez et al. (2017) encontrar una relación espacial con acentuada tendencia al centro-periferia, capitalizando el modelo Airbnb las ventajas que concede la proximidad al centro turístico de la ciudad, cuestión no lograda por los hoteles dadas las restricciones en su desarrollo. 


\section{CUADRO 1}

RESUMEN DE LA LITERATURA ACADÉMICA EMPÍRICA DE EFECTOS DE AIRBNB SOBRE EL MERCADO HOTELERO E INMOBILIARIO

\begin{tabular}{|c|c|l|}
\hline Autor/es & Ámbito geográfico & \multicolumn{1}{|c|}{ Resultados } \\
\hline Fang, Ye y Law (2016) & Idaho (EE. UU.) & $\begin{array}{l}\text { Tras la aparición de Airbnb } \\
\text { aumentó el empleo en el sector } \\
\text { turístico }\end{array}$ \\
\hline Sheppard y Udell (2016) & Nueva York (EE. UU.) & $\begin{array}{l}\text { La presencia de Airbnb incre- } \\
\text { menta los precios de las vivien- } \\
\text { das vendidas entre un 6-11 por } \\
100, \text { y hasta un 32 por 100 (de- } \\
\text { pendiendo de la metodología). }\end{array}$ \\
\hline $\begin{array}{c}\text { Horn y Merante (2017) } \\
\text { (2017) }\end{array}$ & Boston (EE. UU.) & $\begin{array}{l}\text { El aumento de una desviación } \\
\text { estándar en la oferta alojativa } \\
\text { de Airbnb incrementa los pre- } \\
\text { cios de los alquileres un 0,4 por } \\
100 \text { y reduce la oferta alojativa } \\
\text { de alquiler un 5,9 por 100. }\end{array}$ \\
\hline $\begin{array}{c}\text { Barron, Kung y Proserpio } \\
\text { Farronato y Fradkin (2017) }\end{array}$ & Toda la oferta alojativa de \\
Airbnb en EE. UU. & $\begin{array}{l}\text { [Entre otros resultados] El au- } \\
\text { mento de Airbnb en las ciuda- } \\
\text { des explica el 0,27 por 100 de } \\
\text { crecimiento de precios de alqui- } \\
\text { ler y 0,49 por 100 de precios de } \\
\text { venta. }\end{array}$ \\
\hline Zervas, Proserpio y Byers & Texas (EE. UU.) & $\begin{array}{l}\text { [Entre otros resultados] La elas- } \\
\text { ticidad de la oferta de Airbnb } \\
\text { duplica la de los hoteles. Su } \\
\text { presencia reduce ingresos hote- } \\
\text { leros y aumenta el excedente de } \\
\text { los consumidores. }\end{array}$ \\
\hline $\begin{array}{l}\text { [Entre otros resultados] Un au- } \\
\text { mento de la oferta de Airbnb en } \\
\text { un 10 por 100 reduce los ingre- } \\
\text { sos hoteleros un 0,39 por 100. }\end{array}$ \\
\hline
\end{tabular}

FUENTE: Elaboración propia.

\section{Marco legal de las plataformas online de arrendamiento de viviendas en España}

La Constitución española marca el reparto de competencias entre el Estado y las Comunidades Autónomas en materia de regulación de la vivienda turística (artículo 148.1.18). Así, reserva a las Comunidades Autónomas la competencia exclusiva en materia de «promoción y ordenación del turismo en su ámbito territorial». En ejercicio de esta previsión constitucional, todas las Comunidades han aprobado su propia normativa turística. 
Ello ha significado que, tras la exclusión de la regulación de la vivienda turística por la Ley de Arrendamientos urbanos modificada por la Ley 4/2013, de 4 de junio, de medidas de flexibilización y fomento del mercado de alquiler de viviendas, el fenómeno colaborativo en el sector del alojamiento haya pasado a estar regulado por diecisiete ordenamientos distintos. La modificación introducida por el Real Decreto Ley $21 / 2018$, de 14 de diciembre, de medidas urgentes en materia de vivienda y alquiler, que especifica la exclusión del artículo 5.e) de la Ley de Arrendamientos Urbanos, señalando que será de aplicación «su normativa turística sectorial», apenas ha confirmado aquello que ya se estaba aplicando.

Sin embargo, si bien la normativa turística es competencia autonómica, es competencia estatal exclusiva en virtud del artículo 149.1.6 a la legislación mercantil que afecta a la defensa de la competencia, servicios de la sociedad de la información, comercio electrónico y protección de los consumidores y usuarios (Ortuño et al., 2018).

El Estado se reserva también constitucionalmente, artículo 149.1.8, la competencia en la legislación civil (salvo para aquellas Comunidades Autónomas con derechos forales en las materias que posean esos derechos) y la relación contractual entre las partes, así como la planificación general de la economía recogida en el artículo 149.1.13.

Por tanto, la regulación administrativa-turística de las viviendas turísticas queda bajo la regulación autonómica, que se ha venido caracterizando por el carácter restrictivo del fenómeno, mientras que aspectos propios del contenido jurídico obligacional derivado de los contratos, o aspectos en los que resulte de aplicación la normativa mercantil en relación con el sujeto que realiza la actividad, quedan en manos de la regulación estatal y en cuya regulación y actividad, sobre todo a través de los organismos de defensa de la competencia, se han decantado más por la apertura del mercado de la vivienda turística a estas plataformas.

En el Cuadro 2 se recoge una síntesis de la legislación autonómica en la actualidad (diciembre de 2018), que da idea de la dispersión legislativa y criterios aplicados.

Con este escenario legislativo básico, determinados agentes sociales así como instituciones, entre las cuales se encuentra la patronal Exceltur (institución formada por 25 de las principales empresas españolas en turismo y con presencia internacional), consideran que existen una serie de externalidades negativas que se habrían de mitigar y que emanan del masivo volumen de capacidad que las viviendas dedicadas al alquiler turístico han alcanzado en algunos de los principales destinos españoles, fomentadas por el desarrollo de estas plataformas colaborativas (Exceltur, 2016). Argumentos que se contraponen a los expuestos por la propia Airbnb, que justifica una complementariedad en el consumo ${ }^{1}$.

Sin embargo, no en todos los modelos de desarrollo turístico el efecto es el mismo. Así, en las zonas de mayor atracción turística de las grandes ciudades pueden aparecer externalidades negativas que, sin embargo, pueden llegar a convertirse en

\footnotetext{
${ }^{1}$ https://www.airbnb.com/economic-impact/.
} 


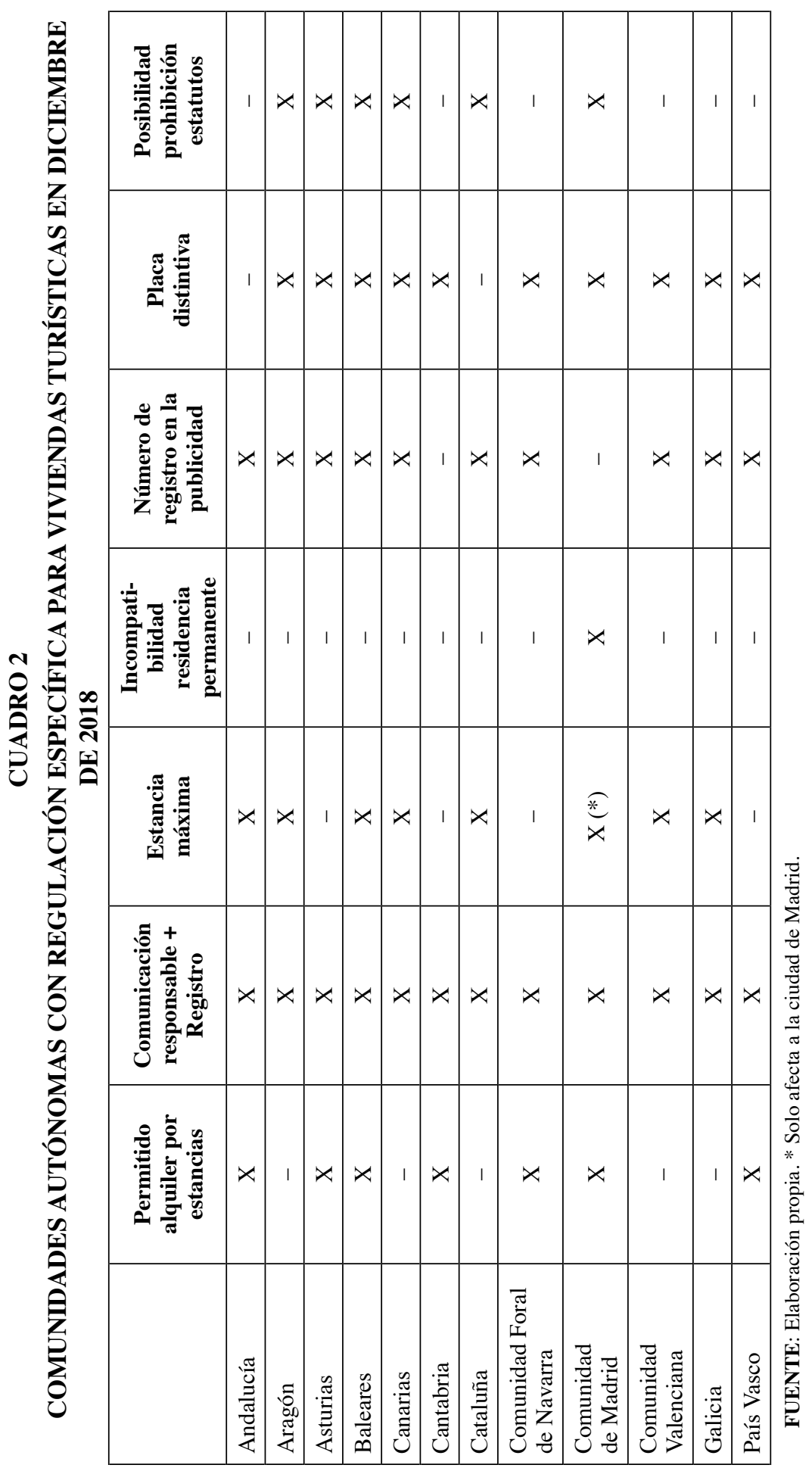


positivas allá donde predomine el turismo residencial de «sol y playa», tal y como afirman Ortuño et al. (2018a). En concreto, detallan las siguientes potenciales externalidades negativas en las grandes ciudades, y su visión contrapuesta en las viviendas de «sol y playa»:

1. Deterioro de la calidad de vida y de los niveles de convivencia. En los destinos de «sol y playa», en realidad se puede producir un aprovechamiento de recursos ociosos, haciendo más sostenible el destino.

2. Tendencia a la desvalorización de los elementos de identidad de la vida de barrio. En destinos que son esencialmente turísticos pero que se «vacían» la mayor parte del año, este fenómeno no produce una sustitución sino una mayor continuidad.

3. Expulsión de la población residente por encarecimiento de la vivienda. Se trata de incrementar el uso de las viviendas en zonas con escasa ocupación fuera del periodo estival, es decir, donde existe un claro excedente de viviendas para ser ocupadas.

Se podría mencionar un efecto adicional, el relacionado con la posible falta de garantía en el cumplimiento de los derechos básicos del consumidor, que sería común a ambos modelos turísticos. Para tratar este último efecto, conviene acudir a la comunicación de Comisión Europea (COM, 2016), donde se señala que «los sistemas de calificación y reputación u otros mecanismos para desalentar el comportamiento nocivo de los participantes en el mercado pueden en algunos casos reducir los riesgos para los consumidores derivados de asimetrías en materia de información. Esto puede contribuir a aumentar la calidad de los servicios y reducir posiblemente la necesidad de determinados elementos reguladores, siempre que pueda confiarse adecuadamente en la calidad de las evaluaciones y las calificaciones».

Con ello se sugiere que la convergencia en la regulación de los alojamientos reglados y las viviendas de uso turístico, se acerque más a una menor intervención administrativa pura de los alojamientos reglados, otorgándole más peso a los sistemas de reputación, que a una hiperregulación de las viviendas de uso turístico.

En definitiva, los diferentes efectos externos según modelos de desarrollo turístico motivan una legislación que debería adaptarse a esa distinta casuística, incluso a nivel de barrios, y todo ello previo diagnóstico que permita el diseño de políticas públicas con evaluación de sus efectos.

Sin embargo, en términos generales se ha puesto el acento en las restricciones al desarrollo del fenómeno en centros urbanos, coartando otros beneficios también generados en esos entornos, como en los casos de Madrid, Barcelona, Valencia o Palma de Mallorca. Estas restricciones se refieren a limitaciones o incluso reducciones de la oferta alojativa, accesos separados a viviendas turísticas respecto del resto de residentes o exclusividad de viviendas turísticas en bajos y primeras plantas, entre otras. 


\section{Desarrollo de Airbnb en España}

En esta sección describiremos la expansión de Airbnb en España a través de los datos facilitados por Airdna, empresa que centraliza y suministra datos sobre la gestión y localización de todos los oferentes en esta plataforma online. A partir de dicha información de origen, hemos configurado una base con periodicidad mensual, conteniendo como unidad de observación el municipio. En concreto, este panel consta de todas las capitales de provincia españolas, incluyendo las ciudades de Ceuta y Melilla, para el periodo 2014-2017.

La entrada de Airbnb en España ha sido relativamente gradual y la información disponible al respecto varía según las ciudades, siendo Madrid y Barcelona las de mayor experiencia en esta materia además de mayores niveles de oferta alojativa en la plataforma Airbnb respecto al resto. Así, se utiliza el número total de camas ofertadas por Airbnb en cada municipio y mes como indicador de esta oferta. No obstante, resaltamos que su cálculo se ha realizado utilizando la oferta disponible un mes determinado según Airdna, pero la agregación de tales valores puede incluir cierto sesgo alcista, al no ser considerados aquellos días en los que el anfitrión bloquea (por los motivos que fuera) el uso de las camas que dispone en la aplicación. En cualquier caso, la tendencia de esta variable es lo relevante.

Dadas las diferencias de tamaño entre provincias, se ha separado la información en cinco niveles, en función de la cuantía en el número de propiedades que estas presentan al final del periodo estudiado (octubre de 2017). En concreto, los niveles en orden de prelación son:

- Nivel 1. Madrid y Barcelona. Ambas superan las 30.000 camas.

- Nivel 2. Entre 10.000 y 30.000 camas: Málaga, Palma de Mallorca, Sevilla y Valencia.

- Nivel 3. Entre 3.000 y 10.000 camas: Alicante, Córdoba, Granada, Las Palmas de Gran Canaria y San Sebastián.

- Nivel 4. Entre 1.000 y 3.000 camas: A Coruña, Almería, Bilbao, Cádiz, Girona, Murcia, Oviedo, Pamplona, Salamanca, Santa Cruz de Tenerife, Santander, Tarragona y Zaragoza.

- Nivel 5. Menos de 1.000 camas: Albacete, Ávila, Badajoz, Burgos, Castellón, Ceuta, Ciudad Real, Cuenca, Cáceres, Guadalajara, Huelva, Huesca, Jaén, León, Lleida, Logroño, Lugo, Melilla, Ourense, Palencia, Pontevedra, Segovia, Soria, Teruel, Toledo, Valladolid, Vitoria y Zamora.

Los gráficos 1 a 5 describen cómo ha sido esta expansión. Respecto a la evolución del número de camas (Gráfico 1), se observa cómo Barcelona dispone en el último mes de la base de datos (octubre 2017) de mayor oferta, concretamente un 58 por 100 superior a la de Madrid. No obstante, la tendencia es claramente positiva en Madrid, en tanto que Barcelona ha tenido varias disminuciones relevantes en el periodo. 


\section{GRÁFICO 1 \\ NÚMERO DE CAMAS DE AIRBNB (AGOSTO 2014-OCTUBRE 2017)}

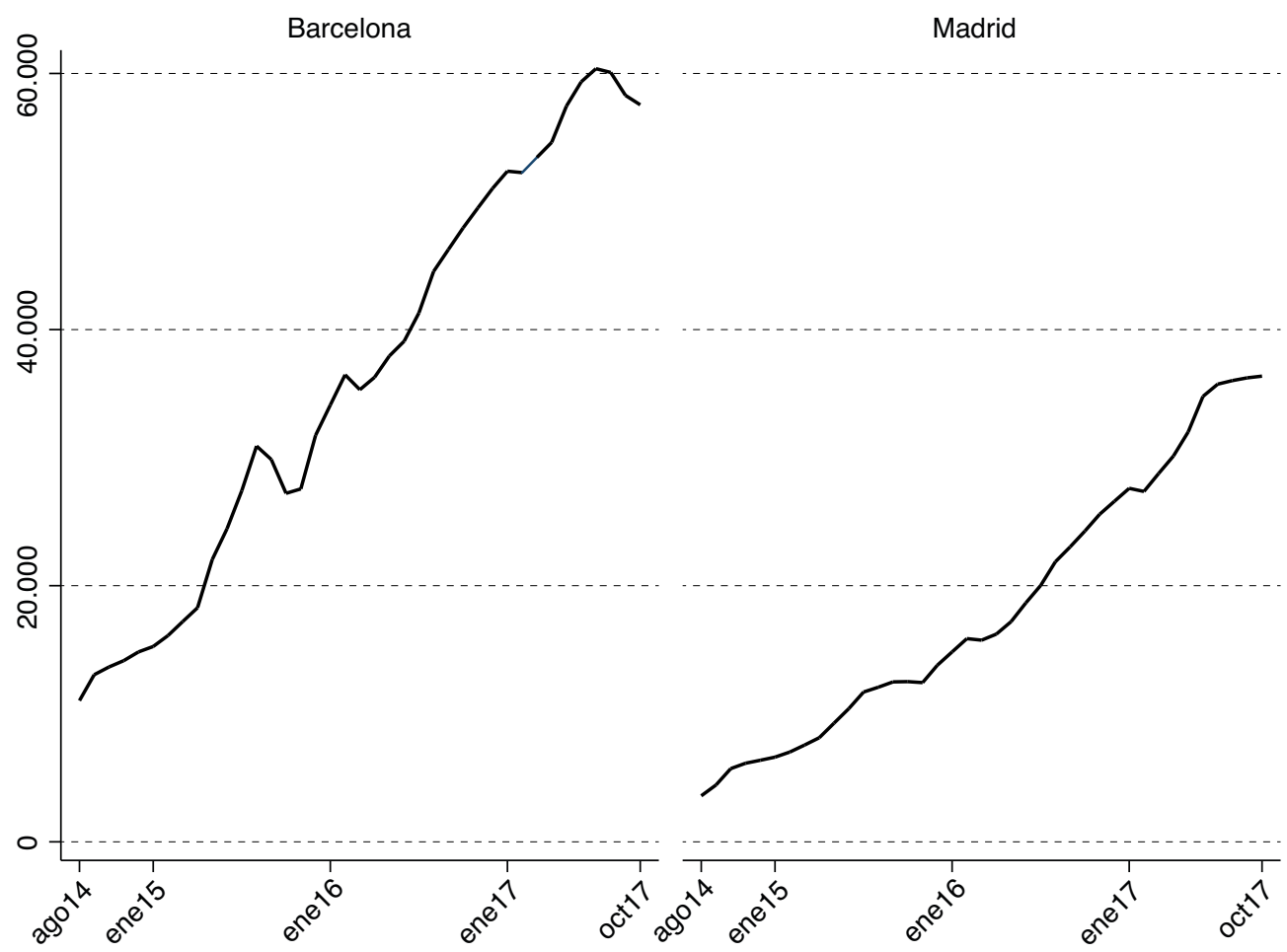

FUENTE: Elaboración propia a partir de información obtenida de Airdna.

En el Gráfico 2 se lleva a cabo el mismo análisis gráfico para el nivel 2. La tendencia en todos ellos es igualmente positiva, aunque se intuye un ligero estancamiento al final del periodo muestral, sobre todo en Palma de Mallorca.

Un tercer grupo de municipios en esta clasificación inicial de oferta, la conforman aquellas situadas entre tres y diez mil camas (Gráfico 3). El comportamiento tendencial en todas ellas es positivo, aunque mucho mayor en Alicante que el resto. En el nivel 4 destacan tendencialmente Pamplona, Cádiz, Bilbao y Santander (Gráfico 4).

Y, por último, las ciudades con menos de mil camas ofertadas, donde son más reseñables aquellas en las que su crecimiento ha sido más moderado que el resto, como son Ceuta, Melilla, Guadalajara, Huesca, Lleida, Palencia, Soria, Teruel y Zamora (Gráfico 5). 


\section{GRÁFICO 2 \\ NÚMERO DE CAMAS OFERTADAS (ENTRE 10.000 Y 30.000) EN AIRBNB}

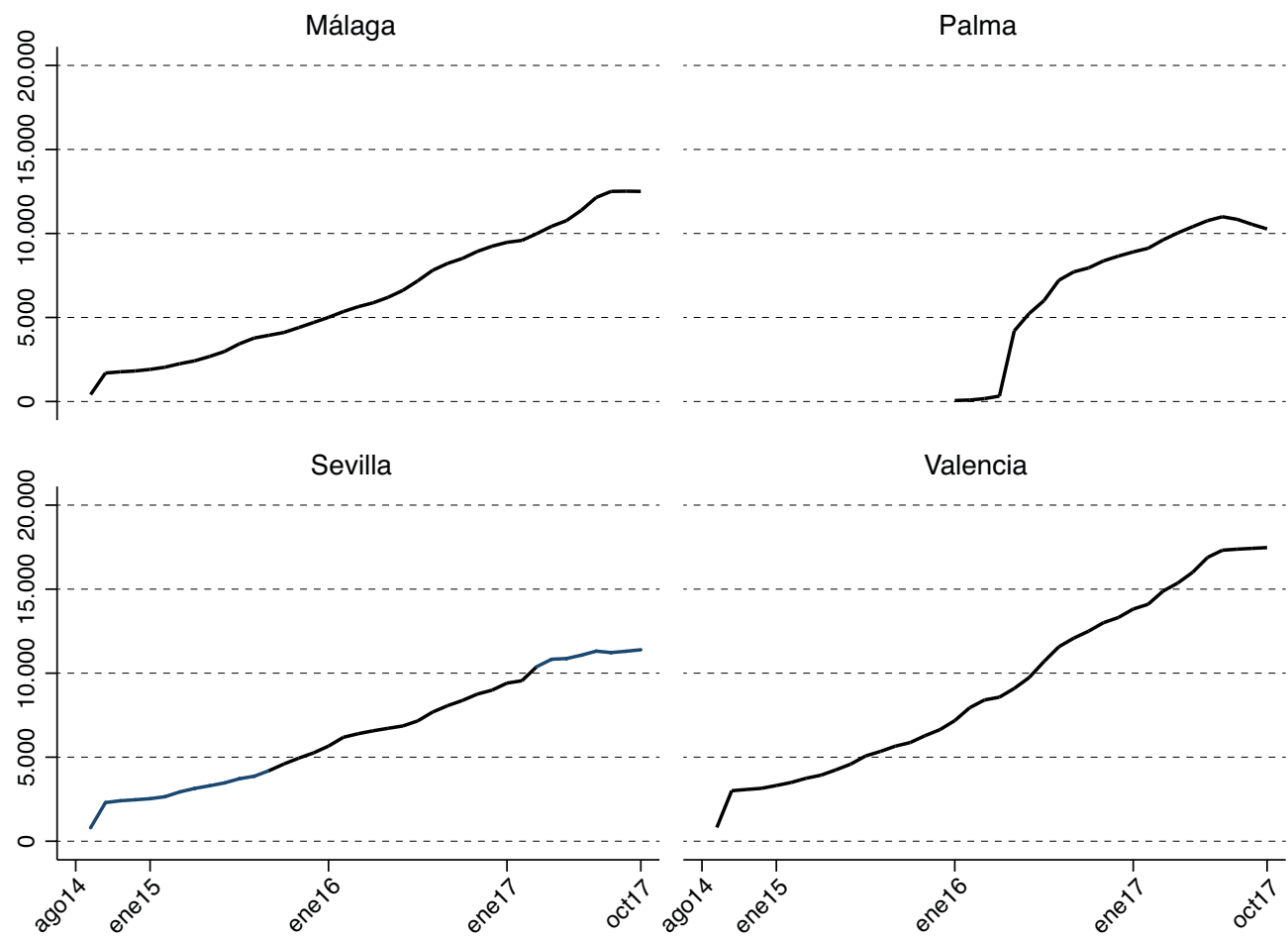

FUENTE: Elaboración propia a partir de información obtenida de Airdna.

Una forma de relativizar esta evolución es dividiendo el número de camas ofertadas por Airbnb en cada capital entre la población de derecho. Aunque estos datos sean anuales permiten mostrar una imagen comparativa entre ciudades. Además, hemos obtenido esta variable relativa en dos momentos del tiempo: abril de 2016 y octubre de 2017. El primero supone el primer mes en el que todas las capitales presentan datos. Por cuestiones de dimensión espacial, excluimos Ceuta, Melilla y el Archipiélago Canario (véanse gráficos 6 y 7). El crecimiento de camas Airbnb per cápita (por cada 10.000 habitantes) es considerable en todas las ciudades, sobre todo en las ciudades de niveles 3 y 4 antes descritas.

Por último, otro indicador de la penetración de Airbnb en las ciudades consistiría en compararlo con la oferta hotelera existente. De esta forma, hemos considerado 


\section{GRÁFICO 3 \\ NÚMERO DE CAMAS OFERTADAS (ENTRE 3.000 Y 10.000) \\ EN AIRBNB}

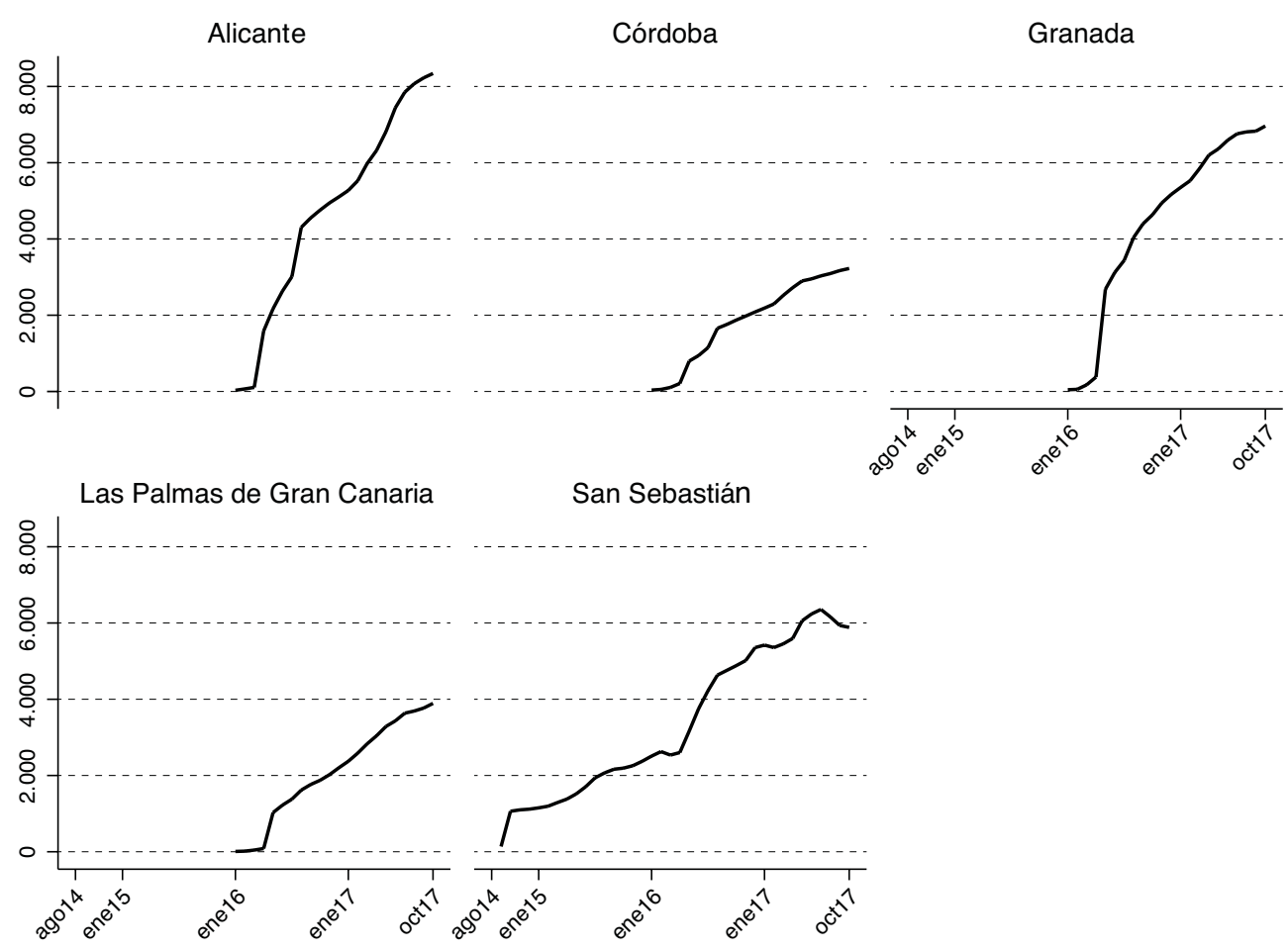

FUENTE: Elaboración propia a partir de información obtenida de Airdna.

solamente las ciudades incluidas en las estadísticas del INE denominadas «puntos turísticos», que se ciñe a 38 ciudades españolas, no necesariamente capitales de provincia.

Como se puede comprobar en el Gráfico 8, los municipios de mayor penetración de Airbnb respecto de la oferta hotelera a finales de 2017 son Málaga (las camas Airbnb son el 121 por 100 de las hoteleras), Alicante (105 por 100) y San Sebastián (103 por 100). En el lado opuesto del ranking se encuentran Badajoz (10 por 100), Teruel $(9,6$ por 100$)$ y Lleida (7 por 100).

Los datos mostrados en este apartado apuntan a un crecimiento importante en esta oferta alojativa en España, con ratios sobre la oferta hotelera existente que llegan incluso a superarla. Evaluar sus efectos sobre el turismo atraído, sobre los propios hoteles o sobre el mercado inmobiliario son líneas de investigación a considerar, tal y como realizan Ortuño y Jiménez (2019). Tales análisis y resultados deberían condicionar las políticas aplicadas al sector. 


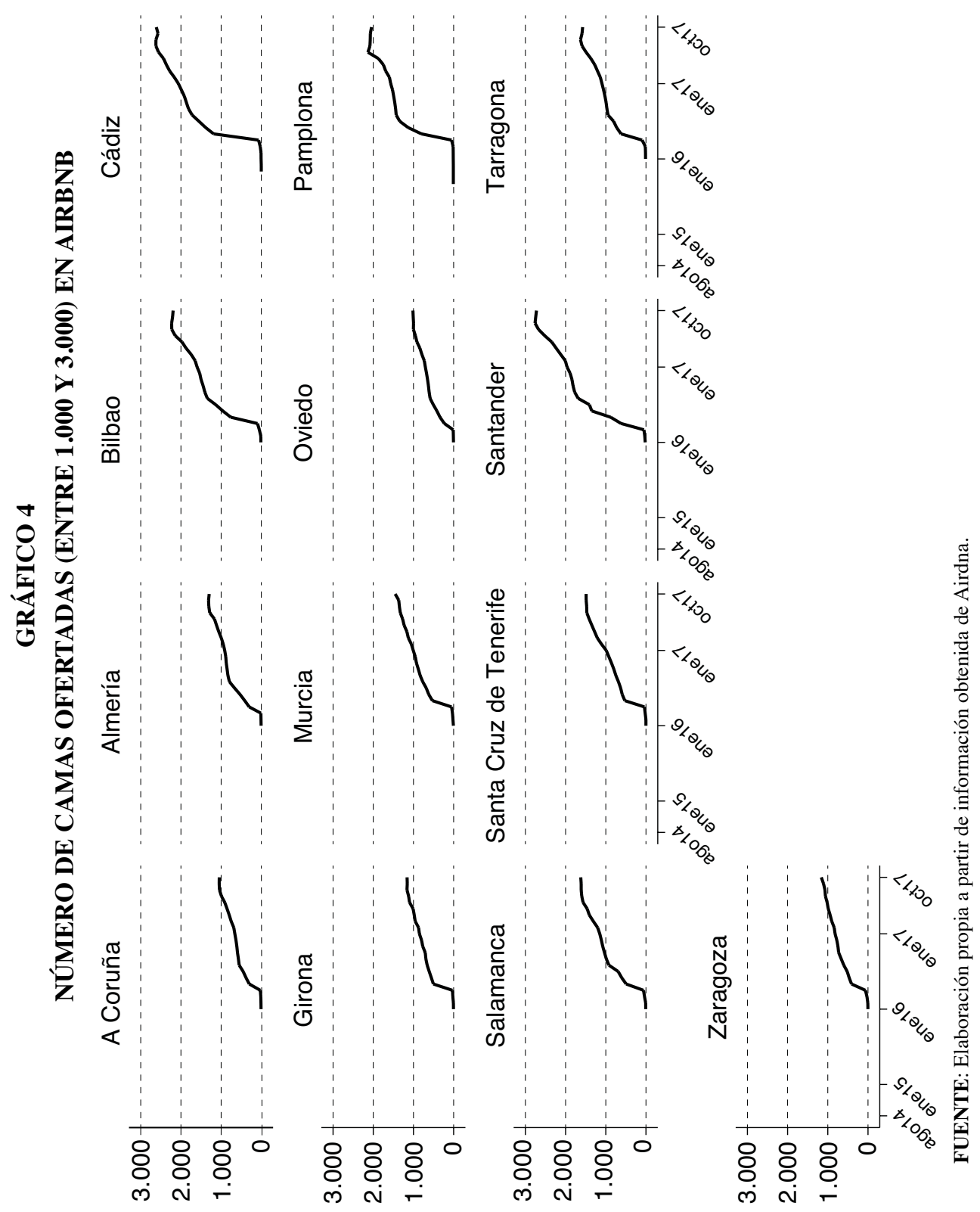




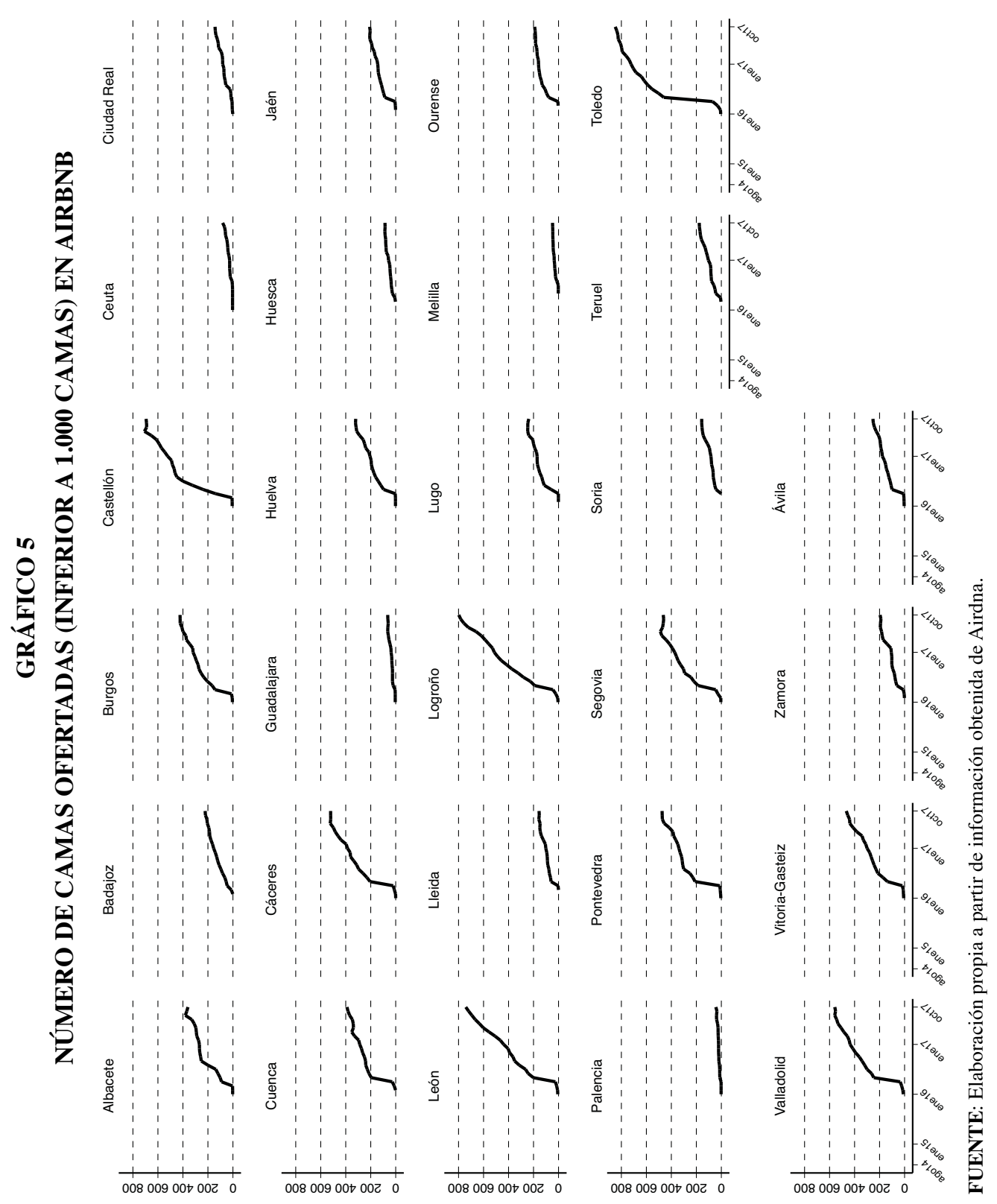



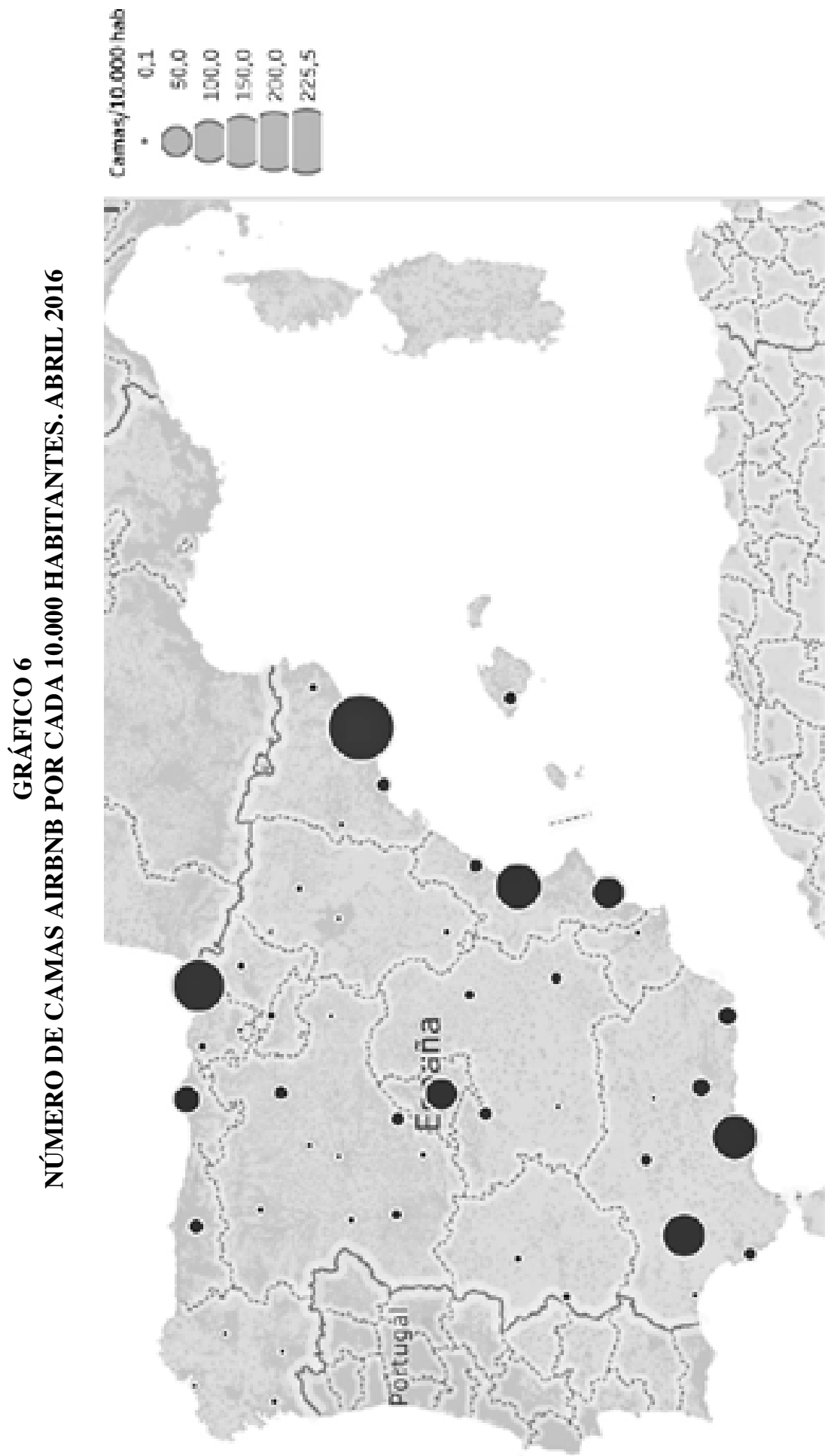

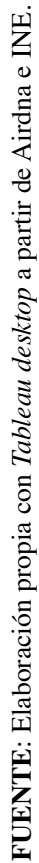



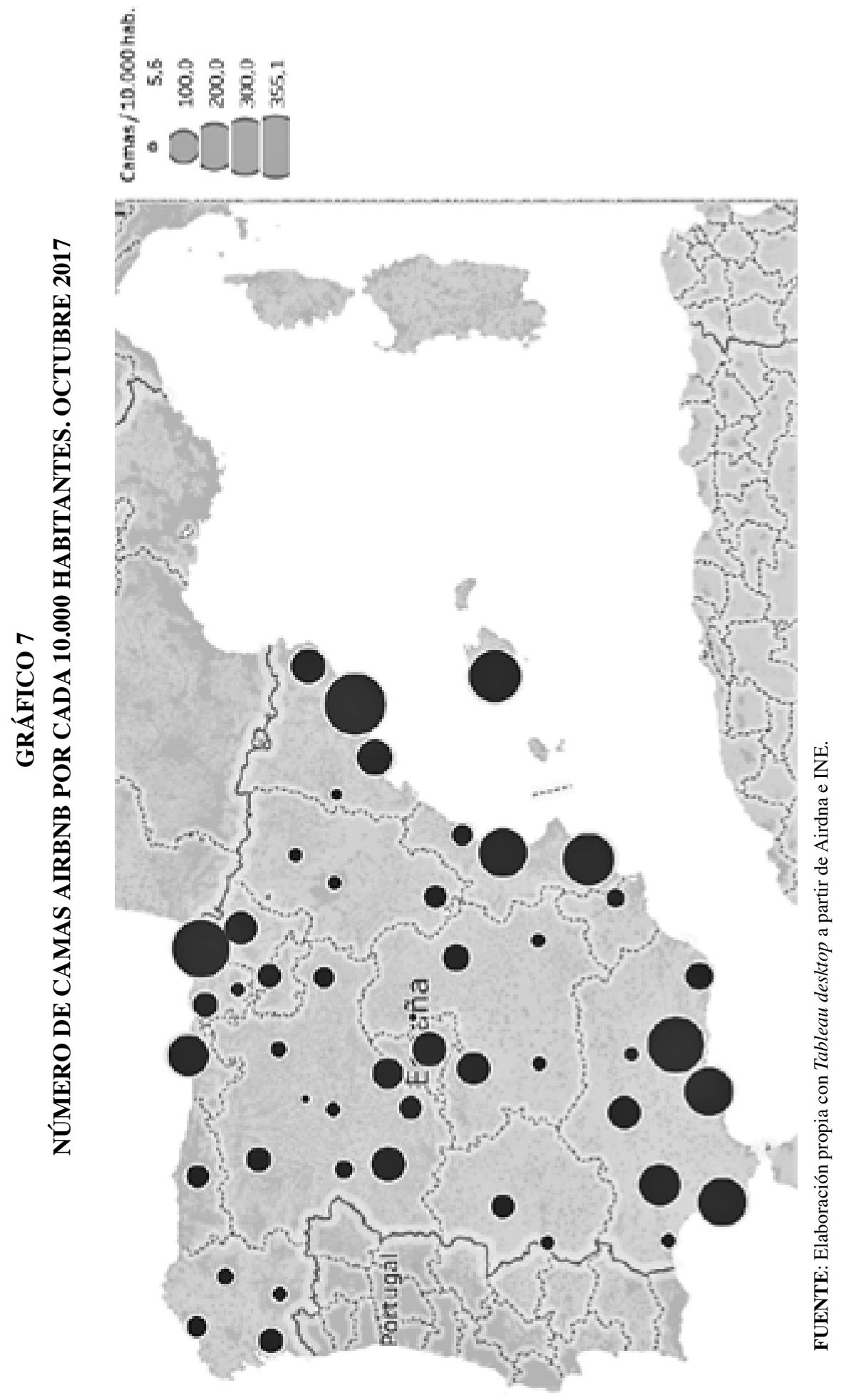


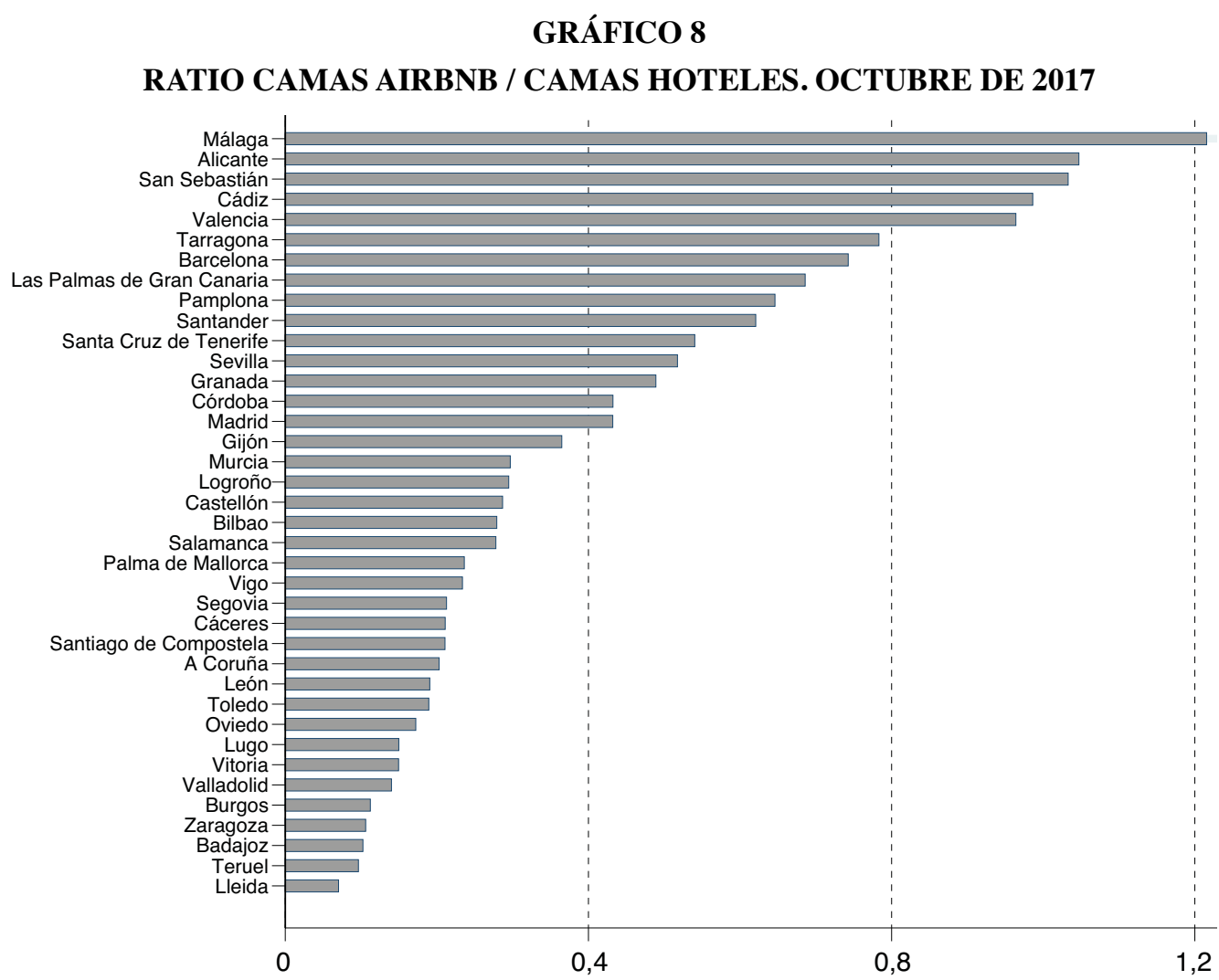

FUENTE: Ortuño y Jiménez (2019).

\section{A modo de conclusión: algunas ideas acerca de cómo gestionar (los efectos de) las plataformas online}

A lo largo de este artículo se ha relatado la entrada de la plataforma Airbnb como oferente de viviendas de uso turístico durante los últimos años y sus efectos sobre distintos modelos turísticos, de manera descriptiva. Así, se han evidenciado las diferentes externalidades generadas según se trate de centros urbanos de gran tamaño o de municipios turísticos de «sol y playa», advirtiendo que la mayor parte de las externalidades negativas en esos centros urbanos pueden transformarse en positivas en zonas residenciales con elevada estacionalidad de «sol y playa».

En estos entornos litorales convendría aprovechar las posibilidades que aporta la economía colaborativa con base tecnológica para que, combinándolas con una propuesta integral de desarrollo urbano y turístico que maximice el uso de los recursos disponibles y de la oferta complementaria, tanto la captación como la gestión del 
turismo captado sean lo más eficientes posible. El turismo de golf, náutico, restauración, oferta cultural y un adecuado uso de los espacios de alto valor ambiental (Ortuño et al., 2018a) puede ayudar a la creación de sinergias positivas en los diferentes sectores, comenzando por el turístico, y, en definitiva, ayudar a la dinamización económica de unos municipios que han dependido en exceso de la construcción de segundas residencias.

Otro de los defectos mencionados en la regulación actual, además de su rigidez, es su dispersión que, si bien puede ser imprescindible en puntos concretos o en zonas concretas, parece adecuado que cuente con una base común protagonizada por el Estado que pueda dar coherencia y simplificar la normativa aplicable. De hecho, la aprobación de la conocida como «Directiva de Servicios» persigue precisamente este fin, al amparo de la cual se promulgan en España dos leyes de singular importancia en esta materia: La ley 17/2009, de 23 de noviembre, sobre el libre acceso a las actividades de servicios y su ejercicio, y la Ley 20/2013, de 9 de diciembre, de Garantía de Unidad de Mercado.

Abundando en esta idea, y tal como señalan Ortuño y Jiménez (2019), este fenómeno que antes de su irrupción se podía regular desde un punto de vista meramente turístico, tiene ahora un impacto multisectorial en vivienda, arrendamientos, revitalización y rehabilitación de centros históricos, turismo, fiscalidad, organización de la economía, urbanismo, libertad de empresa, protección de usuarios y consumidores, protección de la propiedad privada, etc. Todo ello requiere de una mayor coordinación y cooperación desde los tres estamentos competenciales (Estado, Comunidades Autónomas y Ayuntamientos), para garantizar una mayor eficiencia de las políticas públicas respecto de la situación actual y previsible para los próximos años.

De esta forma, el Estado podría establecer las bases de la regulación de las viviendas turísticas sin perjuicio de la necesaria complementariedad de las políticas de urbanismo, de vivienda y turística propias de las Comunidades Autónomas y Ayuntamientos atendiendo a la singularidad de los diferentes municipios, modelos de desarrollo urbano y su problemática asociada (externalidades negativas en centros históricos, zonas de segundas residencias, etc.).

En este marco, con el objetivo de flexibilizar la regulación y que pueda adaptarse en cada momento a las necesidades de ciudades y barrios mitigando las externalidades negativas y aprovechando las positivas, se propone la creación (como un claro second best) de un «Mercado de derechos de arrendamiento de vivienda a corto plazo» (TSR, Transfer Sharing Rights) frente a la aplicación de las medidas restrictivas que se están imponiendo en las grandes ciudades y que, como se señaló previamente, pueden eliminar la mayor parte de los posibles beneficios socioeconómicos que también acarrea el nuevo fenómeno.

Este mercado está basado en el concepto de las TDR (transferencias de derechos de aprovechamiento) aplicado en Estados Unidos, similar al contemplado en el sistema urbanístico español a través de las TAU (transferencias de aprovechamiento urbanístico) y con base en el estudio de Miller (2015). 
En resumen, se trataría de proporcionar a cada vivienda del municipio un derecho de alquiler a corto plazo transmisible y con características distintas según zona o barrio, por un periodo de tiempo determinado. Este sistema podría desarrollarse de la siguiente manera:

- Asignación de TSR. El plazo de un TSR debería ser distinto dependiendo del mercado turístico en cada ciudad o barrio, por ejemplo, limitando el número de personas, de días permitidos, etc., a través de la zonificación. En las nuevas construcciones, se asignaría un valor a los TSR de las nuevas viviendas que se transmitirían al comprador final de las mismas.

- Evitar manipulaciones. Para evitar manipulaciones, solo se otorgarían derechos TSR a los propietarios de una vivienda para evitar que una sola persona física o jurídica pudiera intervenir decisivamente en el mercado. Es probable que esta propuesta se pudiese ampliar a aquellas personas físicas o jurídicas que no tuvieran más de dos viviendas, lo que permitiría ampliar el mercado de las TSR sin que su comportamiento fuera decisivo en el comportamiento general del mercado TSR.

- Canjes de los TSR. Un TSR no tiene valor intrínseco y no transmite ningún derecho, a menos que se establezca un mercado sobre los mismos regulado por la propia ciudad. El propietario de la vivienda usaría una plataforma de internet controlada por la ciudad -ya existe la tecnología para aplicarlo- para canjear un TSR. Estos TSR deberían proporcionar información que permita a la ciudad mejorar su propuesta para el mercado de alquiler a corto plazo: ubicación de alquiler, duración de la estancia, precio cobrado, número de visitantes, etc. Como resulta lógico, el canje debe requerir el pago de una tasa TSR, que será producto de la monetización de externalidades que surgen de la operación de los mercados de alquiler a corto plazo.

- El mercado TSR. Un mercado TSR permitiría a aquellos que no desean utilizar los TSR venderlos a otras personas que quieran participar en el mercado a corto plazo más allá de su asignación inicial. Para evitar que terceros puedan comprar TSR y manipulen el mercado, solo los propietarios de vivienda podrán comprar TSR. El Mercado de derechos de arrendamientos aporta flexibilidad, zonificación e internalización de costes externos.

- Equilibro entre oferta y demanda interbarrios. Si el mercado TSR está diseñado correctamente, habrá escasez en áreas «receptoras» de alojamientos de corta estancia y un excedente en áreas de «envío» de alojamientos de corta estancia.

- Medios para asegurar el cumplimiento. Fundamentalmente, tres medios servirían para asegurar que este sistema se cumple. El primero es la aplicación de todas aquellas medidas propias de los municipios de resolución de conflictos como, por ejemplo, las mediaciones o los apercibimientos por parte de la administración municipal. El segundo es a través de un sistema de sanciones coercitivas que desalienten el incumplimiento. El tercero es un sistema de incentivos para denunciar las violaciones del sistema, de tal manera que el denunciante 
pueda tener determinadas ventajas de índole económica por su contribución al beneficio social.

Una vez establecido correctamente un mercado de TSR, el ayuntamiento podría utilizar este mercado como una herramienta de desarrollo económico y cohesión social con asignaciones de más TSR a unos barrios frente a otros en función de las temporadas del año, del interés de revitalizar determinados barrios, etc.

Como se observa, este sistema evita, por ejemplo, los problemas relativos a la discriminación sufrida por los residentes, que ya no podrían acceder a ofertar su vivienda como turística bajo la prolija regulación municipal que se está aprobando en tantas ciudades, o el beneficio en forma de situación monopolística para los incumbentes, y además posibilita la internacionalización de los costes externos o el desvío de la demanda de alojamiento hacia zonas menos requeridas turísticamente, lo que favorecería la cohesión social y espacial a nivel del conjunto de la ciudad.

De manera muy sintética, se trata de la aplicación dinámica de un sistema de zonificación en que se internalicen los costes externos negativos mediante su tarificación. Puesto que se trata de una propuesta innovadora, se propone su puesta en marcha mediante un proyecto piloto en un entorno urbano acotado que sirva como experiencia y palanca para su exportación a ámbitos territoriales superiores, y siempre que este sea considerado como un mecanismo de mejor resultado potencial que la regulación estricta que reduce las potencialidades del desarrollo de las plataformas online.

En conclusión, España, como gran potencia turística, debería aspirar a convertirse en ejemplo de gestión turística de este fenómeno, lo que requeriría de análisis y estudios que permitiesen la formulación de sistemas de gestión innovadores y basados en las ventajas de la digitalización frente a la aplicación de medidas regulatorias convencionales con dudosos resultados favorables para el interés general.

\section{Referencias bibliográficas}

Barron, K., Kung, E., \& Proserpio, D. (2017). The sharing economy and housing affordability: evidence from Airbnb. Recuperado de https://ssrn.com/abstract=3006832.

Centre for Global Enterprise (2016). The rise of the platform Enterprise. A global survey. Recuperado de https://www.thecge.net/app/uploads/2016/01/PDF-WEB-Platform-Survey _01_12.pdf.

Comunicación de la Comisión al Parlamento Europeo, al Consejo, al Comité Económico y Social Europeo y al Comité de las Regiones. Una Agenda Europea para la economía colaborativa (European agenda for the collaborative economy - supporting analysis). SWD (2016) 184 final, Bruselas, 2.6.2016 COM (2016) 356 final.

Eugenio-Martín, J. L., Cazorla-Artiles, J. M., \& González-Martel, C. (2019). On the determinants of Airbnb location and its spatial distribution. Tourism Economics, 1-21.

Fang, B., Ye, Q., \& Law, R. (2016). Effect of sharing economy on tourism industry employment. Annals of Tourism Research, 57, 234-278. 
Farronato, C., \& Fradkin, A. (2017). The welfare effects of peer entry in the accommodation market: the case of Airbnb (Mimeo).

Gutiérrez, J., García-Palomares, J. C., Romanillos, G., \& Salas-Olmedo, M.H. (2017). The eruption of Airbnb in tourist cities: comparing spatial patterns of hotels and peer-to-peer accommodation in Barcelona. Tourism Management, 62, 278-291.

Horn, K., \& Merante, M. (2017). Is home sharing driving up rents? Evidence from Airbnb in Boston. Journal of Housing Economics, 38, 14-24.

Jimeno I., Aledo, A., \& Ortuño, A. (2018). Airbnb on the Costa Blanca. Diagnosis and proposal of social and tourist integration. International Journal of Sustainable Development and Planning, 13(7), 1008-1019.

Miller, S. R. (2015). First principles for regulating the sharing economy. Harvard Journal on Legislation.

Ortuño, A., Aledo, A., Casares, J., Climent, E., Fernández, N., Jimeno I., Moreno, F. \& Ortuño, R. (2018a). Diagnóstico de potencialidades plataformas $P 2 P$ de alquiler de uso turístico. Municipios turístico-residenciales de la Costa Blanca. Excma. Diputación Provincial de Alicante.

Ortuño, A., Iborra, V., Serrano, S., \& Fernández, J. (2018b). Una primera aproximación al impacto de la plataforma Airbnb en la localización del alojamiento de larga duración en la ciudad de Benidorm. En T. M. Mazón (Ed.), Turismo residencial. Nuevos estilos de vida: de turistas a residentes (pp. 317-332). Universidad de Alicante: Ponencias IV Congreso Internacional de Turismo.

Ortuño, A., \& Jiménez, J. L. (2019). Las viviendas turísticas ofertadas por plataformas online: estado de la cuestión. Documento de Trabajo FEDEA, 2019/4.

Ortuño, A., \& Moreno, F. (2018). Las viviendas de uso turístico, impactos urbanos y propuestas regulatorias (Mimeo).

Sheppard, S., \& Udell, A. (2016). Do Airbnb properties affect house prices? (Mimeo).

Zervas, G., Proserpio, D., \& Byers, J. W. (2017). The rise of the sharing economy: estimating the impact of Airbnb on the hotel industry. Journal of Marketing Research, 54(5), 687705 . 